\title{
Results of Leprosy Control Project, Malawi
}

\author{
B. DAVID MOLESWORTH
}

Lepra Control Project, Malawi

\begin{abstract}
An intensive Leprosy Control Project in Southern Malawi. Using standard dapsone therapy, the effect on the bacilliferous cases was assessed and has produced a very considerable impact.

From the figures it is obvious the time factor is long and, therefore, any shortening of this period, practical for mass campaigns, would be of the greatest importance.
\end{abstract}

From 1966 to 1973 LEPRA has undertaken an intensive Control Project in part of Southern Malawi with a population of 1.3 million in an area of about 2000 square miles lying $16^{\circ}$ South latitude.

The leprosy prevalence is in the region of 15-20 per thousand. By the end of 1973, 13,000 cases had been recorded, some had already received varying amounts of dapsone treatment, the majority had received none (Fig. 1).

Treatment was on an out-patient basis, with weekly visits and the patient given 7 days supply. Once confidence was well established, this was extended to fortnightly, or, in some difficult areas, monthly visits.

Dapsone was the routine drug used in doses of $100 \mathrm{mg}$ daily, reduced to $25 \mathrm{mg}$ daily in 1969. A few cases had a thiozemicarbazone/isoniazid tablet in addition if their MI or BI appeared to be "sticking".

Attendance overall is $50 \%$ and Ellard has shown that, nearly all the dapsone issued is consumed by this $50 \%$ of regular attenders. Defaulting is as much a disease as leprosy and a persistent defaulter remains so in spite of costly and time consuming measures. Persistant defaulters account for $66 \%$ of those cases showing no response or becoming worse.

For social reasons, many of this group are in the 20-40 year olds.

The object of this paper is to show the impact of this intensive control programme, and to this end, the bacilliferous cases have been selected as giving a precise measurement.

The laboratory records of 2280 bacilliferous cases, lepromatous, borderline, and a few tuberculoids in reaction show that:

$76 \%$ become negative.

$13 \%$ show the expected improvement.

$11 \%$ showed no improvement or became worse, indicated by a rise in BI or MI.

Solid forms were still present in $7 \%$ of cases, that is 154 cases, and of these 68 were in the most recent group with less than 3 years' treatment. 


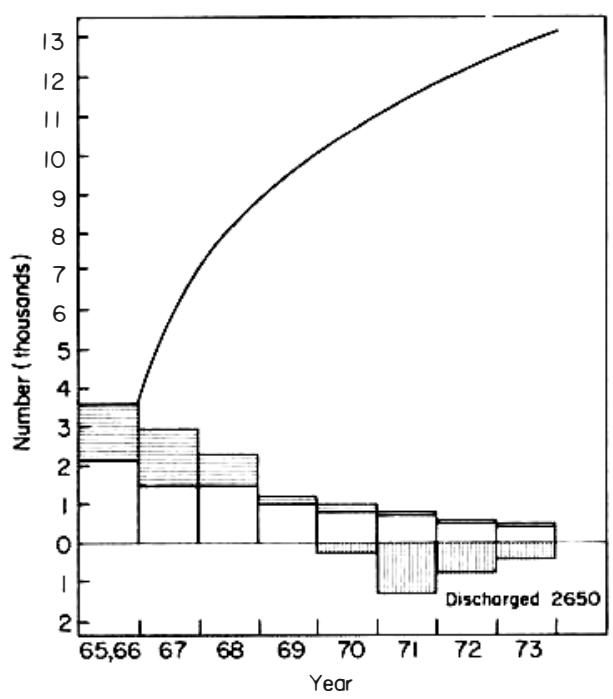

Fig. 1. Registered patients, 1965-1973. 亲 some previous treatment; $\square$ new, untreated; |||||||||| discharged.



Fig. 2. Bacilliferous cases 2280.

As has been stated, two-thirds of the non-improving group are explicable on grounds of insufficient treatment, but one-third are regular and are candidates for other drug regimes or methods of administration.

Figure 1 shows the overall picture with a reduction of case load from 3500 to 500 annually. Untreated cases have fallen from 2100 to 450 , and to discover a partially treated case, apart from those returning from abroad or moving into the area, is now a rarity.

Discharges are nearly all tuberculoid cases and so far, as a matter of policy, no women of childbearing age have been discharged to ensure dapsone cover for pregnancies. 




Fig. 3. Group $\mathrm{BI}<2+, 1724$ cases.

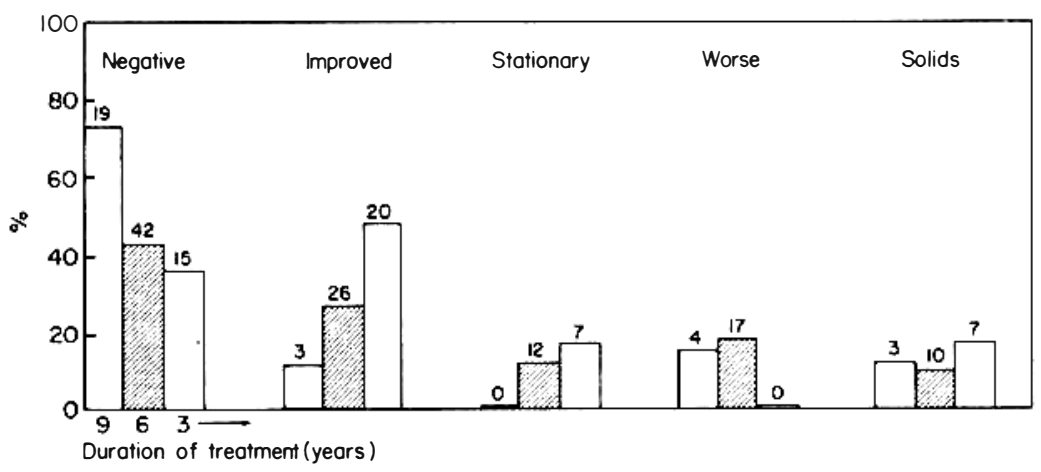

Fig. 4. Group BI 2+, 165 cases.

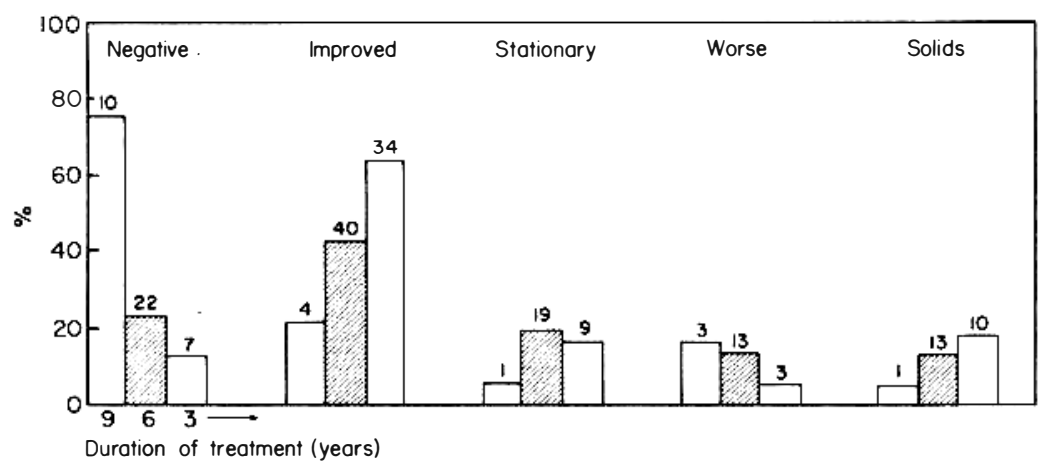

Fig. 5. Group BI3+, 165 cases. 


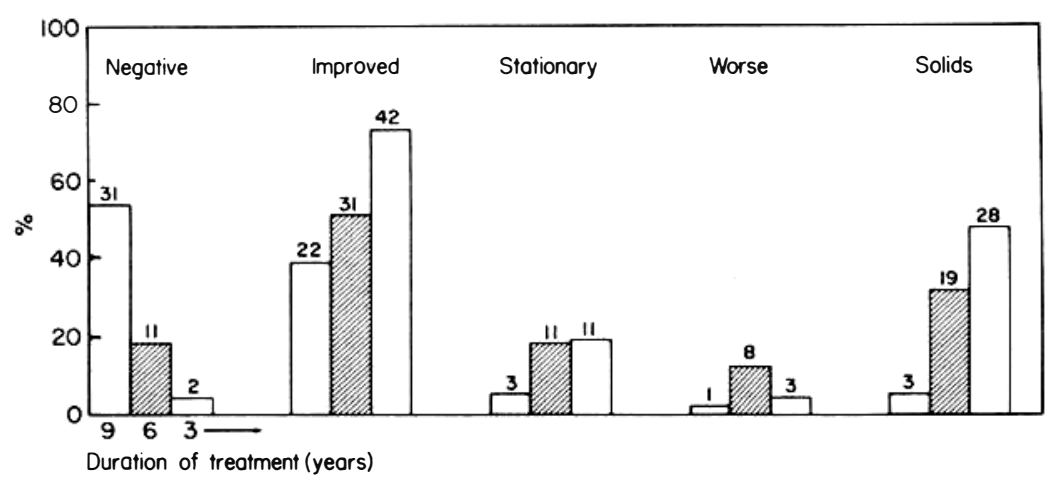

Fig. 6. Group BI4+, 176 cases.

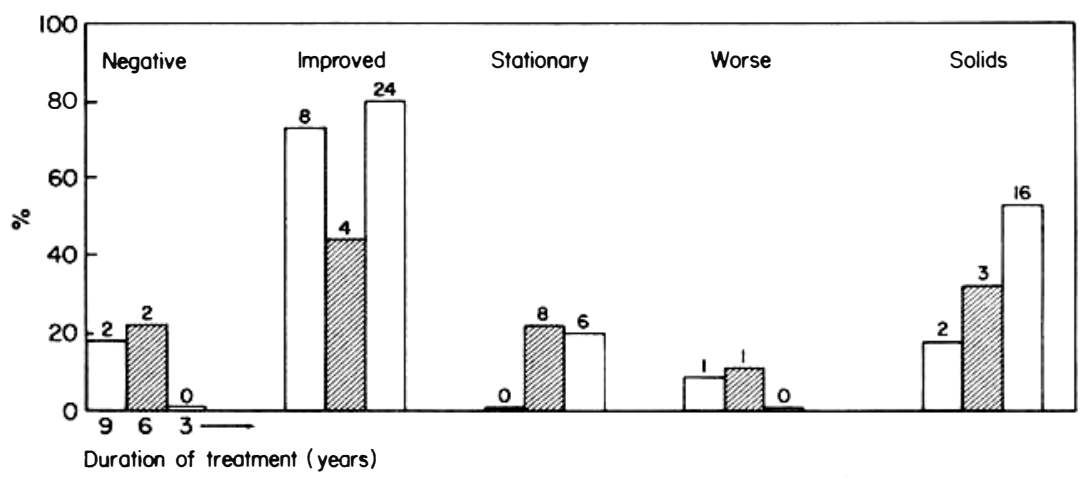

Fig. 7. Groups BI5+ and 6+, 50 cases.

In Fig. 2, of the total of 2280 bacilliferous cases, $76 \%$ show a BI of less than 2 on the Ridley scale. The right hand columns show the overall results obtained.

Cases have been divided into three year groups; the most recent going back to January 1971, 495 cases; the second group back to January 1968, 1486 cases, and the oldest group before December 1967, many of whom had received dapsone before the Project began.

Figures 3-7 show the effect of treatment on groups divided according to BI and duration of treatment.

In the largest group with BI of less than 2, negativity had been achieved in a very high percentage of cases; likewise, since the group contained many old cases who had discontinued treatment, it produces many of the relapses.

The BI and MI are obviously related to the duration of treatment and at the higher levels improvement in BI is far more rapidly obtained than negativity.

In addition to the above figures:

320 cases from beyond our area were transferred for treatment elsewhere;

131 came once and have not been seen since;

150 deaths. 